\title{
Rural Area Development through Multi-interface Technology and Virtual Learning System
}

\author{
Faizan ul Mustafa, Adeel Mushtaq, Shakra Mehak, Salman Akbar, Usman Ahmad, \\ Sara Mobeen, Hassan Ejaz, Tayyab Asif Butt, and Muhammad Faraz Khokhar \\ University of Gujrat, Pakistan \\ faizan_abegmx.com, \\ \{ch.adeelmushtaq, shakramehak, finalyear0, itprof.usman\} @gmail.com, \\ sarah_mobeen@yahoo.com, \\ $\{10050656-091,10050656-115,10050656-021\}$ @uog .edu.pk
}

\begin{abstract}
This paper presents the concept of multi interface technology based on psycho-logical factors of an individual that determine likes and dislikes of a person. According to this concept modern devices will be able to read an individual's likes dislikes and then automatically change their interface including color combination, language, design etc. based on a person's desire. In this paper e-learning concept is also introduced through which we can educate enough to understand a particular technology. After implementing this approach, we will be able to bring people close to the modern technology and can minimize the gap between technology and understanding of a common person living in a backward area.
\end{abstract}

Keywords: Multi interface, smart interface, interface that can automatically change, rural area development, human likes and dislikes and technology.

\section{$1 \quad$ Introduction}

Technology has changed the World. The progress and improvement of technology has improved people's lives. It has led to a far better, easier and very comfortable life for the people. But in rural area of progressing countries especially Pakistan and In-dia, people are still facing many problems in technology adoption. A big part of these countries consists of rural areas especially in Pakistan where 113,678,524 peoples of total population are living in rural areas. Most of them are illiterate people; they don't have knowledge to use a particular technology. That's why people living in rural areas are facing technology emergence.

This paper focuses on the psychological factors that plays major role in a person's liking. For example, according to modern researches, group of people belonging to same blood group, age and birth order, have many common personality traits. They have common likes and dislikes towards a particular design and combination. While color psychology helps us to find personality traits of those people who have same favorite colors. This research paper discusses that how we can make such user interfaces that can automatically change their behavior by fetching the certain personality 
traits of the user. In villages most of people follow old trends, they don't believe on modern healthcare facilities and technology. If we change their thinking level we can bring a massive change in villages in all dimensions. For this, virtual education system can play a vital role. According to this approach, interface should be able to teach user how to interact with technology, how to deal with health related problems, traffic rules etc. in his own native language. This is the virtual learning concept that is discussed in this paper. A villager who works in fields' the whole day and cannot go to school, he can get basic knowledge from the device that is being used by him. Government can run these projects parallel to those that are being executed by them.

In section 2 problem background scenario is discussed, here in this section it is briefly stated that why people feel it difficult to adopt technology instead of old local methods. In section 3 related work is discussed which is based on current research on both psychological factors that affects human like's dislikes and interfaces. In section 4 solution of problem is explained in details. This section includes three parts, in 4.1 overview of solution is given, while in 4.2, Technical details of solution are explained. Section 5, 6, 7 and 8 have expected results and, conclusion respectively.

\section{Problem Background}

With the growing progress in Information technology is offering the potential for improving the life style of the people. Rapid growth in Technology has brought people closer and minimized the class difference among them. After the invention of graphical user interfaces GUI, people's views and perception about the technology has drastically changed. This tremendous change in the technology has deep effects on life style of the people living in the backward areas. Nonetheless, in many progressing countries like India and Pakistan people especially in rural areas are part of worst digital divide. According to the World Bank report of 2012 almost 1.5 billion of the world's poorest people live in rural areas. They have poor infrastructure, a huge gap in communication means, old tradition and living style, lake of living facili-ties like electricity, water and health centers. These rural people supply food to otherworld but majority don't use modern equipment and technology. Another thing that is very important $37.6 \%$ land of rural areas of World and $34.4 \%$ of Pakistan's land is agricultural land [1]. Unfortunately this ratio is becoming less and less day by day because population of these areas is increasing rapidly. This is really dangerous and can affect food availability in World. Governments of all countries are developing roads infrastructure, providing subsidies to farmers but rural areas are still different and backward than other areas.

Technology adoption is a critical problem in these rural areas. Because of the literacy rate that is very low, people don't know how to use the modern technology in their daily life. They are not able even to operate a smart phone that connects them with other World. User interface plays a critical role during the interaction with technology. A user interface provides optimum communication between the user and the device. Although user interfaces are designed to facilitate user to interact with the technology but they are many factors that varies person to person[14]. If an interface 
is good according a person living in USA, at the same time it may be bad for another person living in a village of Subcontinent. So it was needed badly such an interface that should be smart enough to change itself automatically according to an individual's liking. Our cities are advanced because people are educated they can understand their responsibilities well; they can get good facilities than others. But in villages most of people follow old trends, they don't believe on modern healthcare facilities and technology. If we change their thinking level we will bring a massive change in villages in all dimensions. For this virtual education system can play a vital role. So it is need of time to develop such a interfaces which not only automatically change but educate their users too. That's why we have proposed multi-interface technology and virtual learning concept in order to overcome technology adoption problems in rural areas.

\section{Related Work}

Basically Pakistan is an agriculture country. More than two-thirds of Pakistanis live in rural areas, of which about 68 percent are employed in agriculture, agriculture is backbone of Pakistan's .these people provide us food but they didn't have good meal of one time, the rural people are mostly facing poverty. More than 60 percent of poor rural do not have basic facilities that we can imagine our lives without these facilities like water for drink, food, electricity, health center etc. Mostly these poor people do not have educational background that is the main reason that they are facing crisis. .

\subsection{Digital Divide}

Due to the gap between digital and non-digital world, a bridge is created between rural and urban areas, called Digital Divide. There are several major causes to the digital divide including technology emergence for illiterate people. As expected, the people in rural areas have less know how about digital devices. Studies of digital divide often focus on lack of technology. Moves to expand computer availability with simple interfaces will help to increase usability of machines[10]. In order to bridge this Digital Divide, global Organization is making long-term investments to collect and distribute technological devices and teach people how to use technology. Organiza-tion such as the United Nations, World Bank and other not-for - Profit Organization are working toward closing the gap between the digital and non-digital Divide.

\subsection{Psychology Related Review}

There are some biological attribute that influence human behavior. The attribute we have used is blood group. A natural experiment was conducted in japan in 198781988 to examine the relationship between blood groups and human behavior. Japanese people propose a prophecy that blood groups have great influence on human personalities. This phenomena constructed Japanese beliefs that they started to use 
this study in social psychology, educational psychology, clinical psychology Organizational and industrial psychology. They conducted an open personality test where people can participate. There they practice many activities to judge personality. Before this test, they classified them according to their blood groups.

After accomplishment of this study, they analyze the results. At very extent, the persons having same blood groups have same results in activities that had performed during test. Experts note down their personalities qualities then they concluded about blood-typical personality's stereotypes [2]. This topic of blood groups and humans personality is very popular domain. Research is going on upon this topic from a long time ago. In 1964 a research was conducted to study the relationship among human personality and blood groups. A group of people was studied according to their blood groups. Results of the comparison over all 14 HSPQ factors indicated that only factor I reaches a substantial level of significance. Consideration of the adjusted means for the blood group on this factor indicates that highest scores on I are associated with type A, somewhat lower scores with types and B (in that order), and the lowest scores with type $\mathrm{AB}$. Just short of significance at the $5 \%$ level is Factor $\mathrm{J}$, asthenia (the common feature of neurasthenia and psych asthenia), the $\mathrm{AB}$ type being low on this factor. As human's age grows, his likes, dislikes and perception toward things changed [3].

Study was conducted to know the impact of human age on his behavior. They had divided this study into two portions, the first portion is about the typical middle-age adult and the other is about typical young-age adult. The sample of this study was $1,267,218$ people from population between ages of 10-65 years. After testing on sample they had developed a personality mode that was called Big Five traits domains. According to this study, people are:

Extraversion (Quick in decisions, hard work), agreeableness (pleasant personality), Conscientiousness (shy, take time to make decisions), Neuroticism (moody, calm in tension), and openness to experience (defender, use their experience in decision making). This Big-five model planned a test to judge people about their personalities. The available results indicate that from emerging adulthood through middle age, conscientiousness and agreeableness show positive age trends, Neuroticism shows a negative age trend, and Extraversion and openness to experience show flat trend.

These studies provide an informative sketch of age differences in personality traits but there is need to complete this picture so further work is required.[4]

Color has increased the learning efficiency. Color is a kind of factor which has a great impact on human emotions, mind and decisions. This paper has analyzed impact of color factor on human learning. Color is due to a wavelength spectrum of exposure to the human eye, and causes the retina to produce visual excitement by color vision cell [5]. Hue, saturation and lightness are three basic characteristics of color. Color psychology is the subjective psychological reaction caused by objective color world. Learning efficiency is the ratio of quantity and quality for the time, energy consumption and outcomes though studying. It has a good effect for learner, for example light blue color helps to take concentration, and similar color has great impact on human mood and behavior. Pleasant color brought good change in human. In this paper, a color testing was performed on thirty students having same characteristics, to check 
their learning efficiency in different color's environment. It was concluded that the formation of a specific color of color vision psychology, given the character and association of different color. So when people see the different colors will produce the appropriate physiological and psychological changes. This change will turn affect the learner's learning efficiency [6].

According to social and clinical psychology there are many factors that decide human likes and dislikes and general behavior towards a certain event. A study was conducted in 2001 that covers the review of twenty five years of research on human evaluation conditioning [7].

\subsection{HCI Related Review}

Although the details of how people use command-line interfaces are fairly well known that how complex to remind commands to operate machine. Few decades ago, computer experts an introduced graphical user interface (GUI). GUI has brought a tremendous change to adopt technology, but still the problem exist that for a lay person, it is difficult to operate to understand interface of machine, so the sub-field Computer Sciences, Human Computer Interaction (HCI) has been working to overcome this problem. The GUI is based on a hybrid mechanism that combines ranking and rating. It has been used based image for rating. It presents a base image for rating its similarity to seven peripheral images that are simultaneously displayed in a circular layout. The user is asked to report the base image's pair wise similarity to each peripheral image on a fixed scale while preserving the relative ranking among all peripheral images. The collected data are then used to predict the user's subjective opinions regarding the perceptual similarity of images. We tested this new approach against two methods commonly used in perceptual similarity studies: (1) a ranking method that presents triplets of images for selecting the image pair with the highest internal similarity and (2) a rating method that presents pairs of images for rating their relative similarity on a fixed scale. We aimed to determine which data collection method was the most time efficient and effective for predicting a user's perceptual opinions regarding the similarity of mammographic masses. Our study was conducted with eight individuals. By using the proposed GUI, we were able to derive individual perceptual similarity profiles with a prediction accuracy ranging from $76.83 \%$ to $92.06 \%$ which was $41.4 \%$ to $46.9 \%$ more accurate than those derived with the other two data collection GUIs. The accuracy improvement was statistically significant [8].

To overcome a gap between current representational capabilities[11][12][13][14] [15][16] of cognitive models and the requirements for cognitive modeling in humancomputer interaction a research was conducted that proposed that introduced the understanding of human-computer interfaces as devices and a form of representation where information is exchanged between an artificial and natural representation system, in order to accomplish tasks cooperatively. The representations of the computer system and the cognitive system are mutually modulating and influencing each other. The goal is to establish a process of cooperative human-machine cognition [9]. 
Similarly in 2009 a research was conducted in which various interaction models are explored and investigated when used in designing interactions. Object Action Interface model is studied in detail and its shortcomings are identified [10].

\section{$4 \quad$ Proposed Solution}

Majority of people who belong to rural areas are illiterate. Main problem that an user interfaces while interacting with software embedded devices is to understand the inter-face. Although software developers try their level best to minimize this gap but $100 \%$ perfection is not possible. A common lay man who uses the technology faces many problems during his interaction with device.

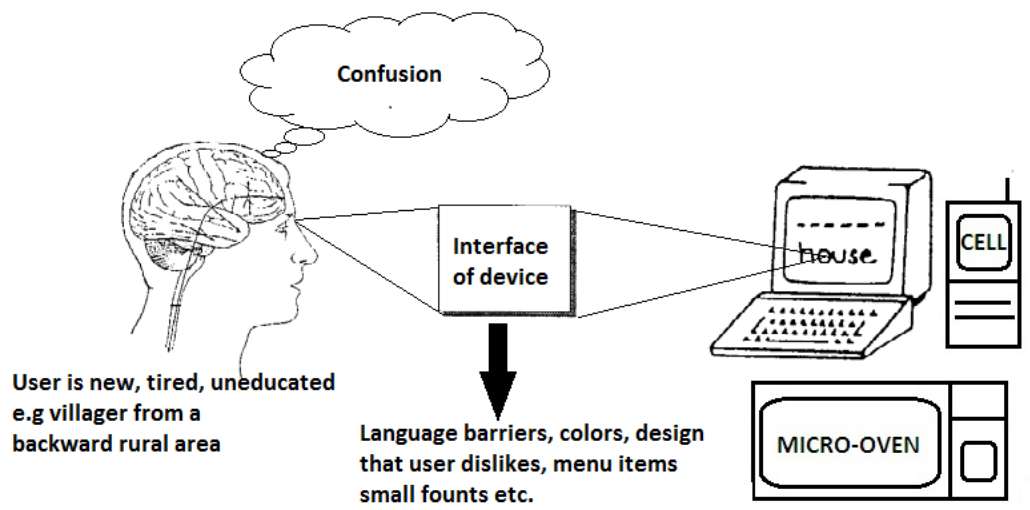

(Major problem in rural areas while using software embedded devices)

Fig. 1. Device usage issues

\subsection{Overview of Solution}

We have proposed multi interfaces technology concept to overcome technology adoption gap. Parallel to this, for enhancing user's literacy level virtual learning concept has been introduced. According to this concept, the device that is being used by a non-technical person will get some information about user. This data will be collected by user with the help of a simple questionnaire before using a device. This information will include following points

- Age of user

- Blood group of user

- Favorite color

- Birth order of the user

- User's Education

- Area 
According to psychology above mentioned information has deep concern with an individual's likes and dislikes. By analyzing above mentioned information with the help of psychological theories we can predict following points about user

- Education level

- User's language

- Color combination that user likes

- Design pattern that user likes

After analyzing this information device will search most suitable interface pattern from its built-in memory and will generate a view in user's native language according to user's preferences. This will help user to use a particular device more easily.

\subsection{Technical Approach}

In technically language this research has been divided into three steps which are listed bellow as:

- User Authentication

- Machine Response

- System Updating

The whole process is described through following figure.

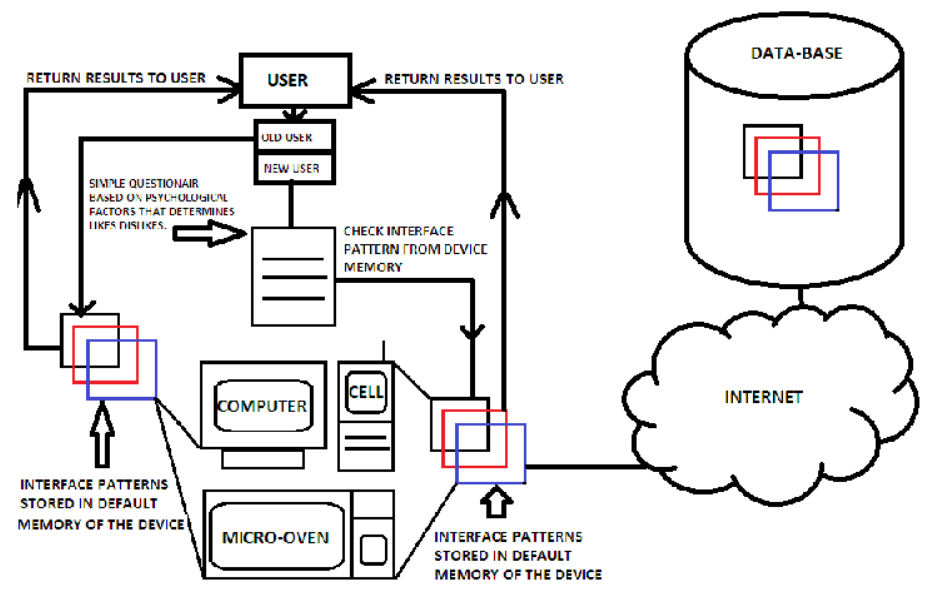

(HOW A MULTI-INTERFACE APPROCH WORK)

Fig. 2. Technical model for the process

User Authentication. Whenever a user will interact with the device, device will ask him to confirm whether user is old or new. If the user is new it means that he is using 
the device first time then the device will ask user to provide some information about him through a simple questionnaire. Questionnaire will have following questions

- Age of user

- Blood group of user

- Favorite color

- Birth order of the user

- User's Education

- Area

After getting this information device will allocate user, user ID number and will allow user to use the device. In case of old user machine will require only user's ID and precede the next process.

Machine Response. We are assuming that our devices are smart enough and have built in memory with it. All possible interface patterns are stored in device's memory based on the questionnaire. After data collection from the new user, device will analyze data and match this information with the interface patterns stored in default memory of device. If the pattern is found, device will change its interface according to that pattern.

If the pattern is not found, device will return interface pattern close to user's information and call the database to send interface patterns of based on user's information so that when user comes second time device will allocate new interface.

System Updating. Although devices will have the entire possible interface patterns stored in their default memory but exceptions are still possible. If a device does not find a pattern related to users information, it will store user's info in its memory and will make a connection with main database to update its record with new patterns. User can also update his device with new interface pattern whenever he wants.

Virtual Learning. Virtual education system can play a vital role. According to this approach, interface should be able to teach user how to interact with technology, how to deal with health related problems, traffic rules etc. in his own native language. This is the virtual learning concept that is discussed in this paper. A villager who works in fields' the whole day and cannot go to school, he can get basic knowledge from the device in his native language that is being used by him. Government can run these projects parallel to those that are being executed by them.

Technical this idea runs similar as mentioned above in figure number 2 . 


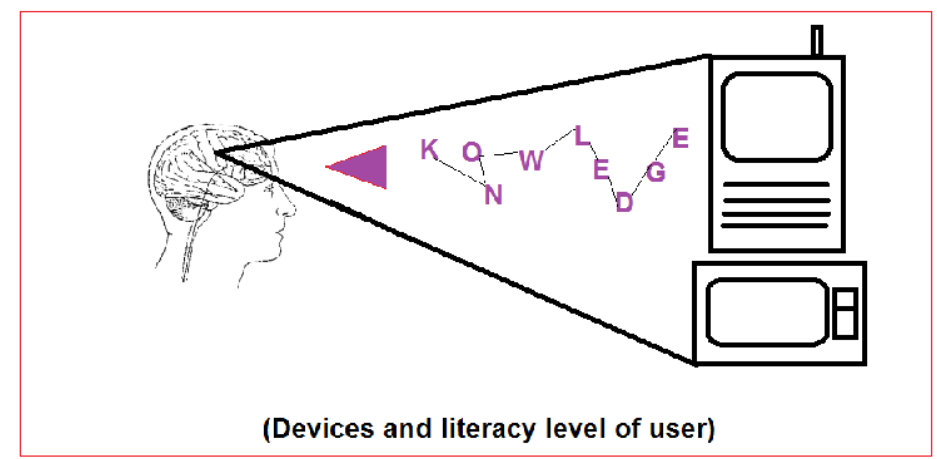

Fig. 3. Virtual learning model

\section{$5 \quad$ Expected Results}

In future, we will implement this idea and the expected result according to our research will increase use of technology in rural areas. People belonging to backward areas will have confidence in technology devices. There will be a chance to control digital dived that has divided a world into different parts. There are following benefits of overcoming digital divide that will be seen when this idea will be implemented, these are:

1. Those without enhanced data capability will be able to access the benefits expected particularly in relation to education, health and government services.

2. Concern that people in the developing countries will enjoy the benefits of the new knowledge-based economy using technology.

3. It will remove disempowers, discriminates and dependency, and reshape the map of the world.

4. If people adopt technology easily, it is solution for traffic and fuel pollution

5. Key to establishing availability, accessibility and affordability for people.

\section{Conclusion}

In this study, various human attributes are explored and investigated to understand human personality and machine interface Designing. These attributes are age of user; blood groups, color, user education etc. proposed model is represented in the form of set of finite interfaces that are designed according to mentioned attributes. As user fulfill the form requirement, the system will response and machine interface will be changed according to person's psychological attributes.

The implementation of a set of infinite interfaces based on this proposed paradigm is the future work. We will also evaluate developed interfaces using qualitative usability evolution measurement methodologies. 


\section{References}

1. World Bank, http: / / data.worldbank.org/indicator/SP.RUR. TOTL

2. Sakamoto, A., Yamazaki, K.: Blood-typical Personality Stereotypes and Self-fulfilling Prophecy (April 2002)

3. Cattell, R.B., Boutourline Young, H.: Blood groups and personality traits. Supported by public health grant MH 01733-17 by Grant Foundation of New York (received February 17, 1964)

4. Soto, C.J., John, O.P., Gosling, S.D., Potter, J.: Age Differences in Personality Traits From 10 to 65:Big Five Domains and Facets in a Large Cross-Sectional Sample. Journal of Personality and Social Psychology 100(2), 330-348 (2011)

5. Cigić, D., Bugarski, V.: Personality traits and color preferences, Research Article UDC 159.937.51

6. Hartman, T.: The Power of Color: What Does Your Color Say About You. Colorcode.com

7. Houwer, J.D., Thomas, S., Baeyens, F.: Associate learning of likes and dislikes. Psychological Bulletin 127(6), 853-869 (2011), copyright 2001 by American psychological association, Inc., 0033-2909/01/\$5.00, doi:1037///0033-2909.127.6.853

8. Seneler, C.O., Tugrul, N.B.: A Taxonomy for Technology Adoption: A Human Computer Interaction Perspective. In: PICMET 2008 Proceedings, Cape Town, South Africa, PICMET (July 27-31, 2008)

9. Markus, F., Peschl, Stary, C.: The Role of Cognitive Modeling for User Interface Design Representations: An Epistemological Analysis of Knowledge Engineering in the Context of Human-Computer Interaction, ICPC: PIPS No.: 160298 (mind:mindkap.cls) v.1.15 mind287.tex; 11:57 (October 21, 1998)

10. Rashid, U., Niaz, I.A., Amin, M.W., Bhatti, M.A.: Designing Interactions using OAI Model: A New Interface Modeling Paradigm. In: International Conference on Emerging Technologies (2009)

11. Sheikh, J.A., Fields, B., Duncker, E.: Cultural representation by Card Sorting. Ergonomics for All: Celebrating PPCOE's 20 years of Excellence. Selected Papers of the Pan-Pacific Conference on Ergonomics, November 7-10, pp. 215-220. Taiwan CRC Press, Kaohsiung (2011a)

12. Sheikh, J.A., Fields, B., Duncker, E.: Multi-Culture Interaction Design. In: Advances in Cross-Cultural Decision Making, pp. 406-415. CRC Press (2010b)

13. Sheikh, J.A., Fields, B., Duncker, E.: Cultural based e-Health information system. Presentation at the Health Libraries Group Conference 2010: CILIP, Salford Quays, UK, July 1920 (2010a)

14. Sheikh, J.A., Fields, B., Duncker, E.: Cultural Representation for Interactive Information system. In: Proceedings of the 2009 International Conference on the Current Trends in Information Technology, Dubai $(2009 \mathrm{c})$

15. Sheikh, J.A., Fields, B., Duncker, E.: Cultural Representation for Multi-culture Interaction Design. In: Aykin, N. (ed.) IDGD 2009. LNCS, vol. 5623, pp. 99-107. Springer, Heidelberg (2009b) 